\title{
Development of a Commercial Laboratory Scale Soft X-ray Microscope
}

Tony McEnroe ${ }^{1}$, Fergal O'Reilly ${ }^{1,2}$, Paul Sheridan ${ }^{1}$, Jason Howard ${ }^{1}$, Ronan Byrne ${ }^{1}$, Aodh O’Connor ${ }^{1}$, David Rogers ${ }^{1}$, Ciaran Rogers ${ }^{1}$, Conor McCarthy ${ }^{1}$, Alberto Manzoni ${ }^{1}$, Dunja Skoko ${ }^{1}$ and Kenneth Fahy ${ }^{1}$

${ }^{1 .}$ SiriusXT Ltd., Science Centre North, Belfield, Dublin 4, Ireland.

2. School of Physics, UCD, Belfield, Dublin 4, Ireland.

Cryo-soft X-ray tomography (cryo-SXT) is an extremely powerful technique that allows the imaging of an entire cell in its fully hydrated state with natural contrast. Whole cells up to 10-15 microns thick can be imaged at a 3D resolution approaching $30 \mathrm{~nm}$. Cryo-SXT preserves volatile structures, and since the cell is fully hydrated, avoids artefacts associated with sample shrinkage during dehydration. Cryo-SXT can also image the thickest parts of the cell, including the perinuclear region that contains many of the cell's organelles, which cannot be imaged in 3D by other techniques. Great progress has been made over the last decade in developing cryo-SXT as an imaging technique on synchrotron hosted microscopes [14]. Workflows have improved which allow non-synchrotron researchers to access the technique, and significant expertise has been developed in correlating SXT and cryo fluorescence data [5-7]. This amalgamation of techniques integrates 3D molecular localisation data with a high-resolution, 3D reconstruction of the cell. Here we report on the development of a compact lab based microscope that aims to deliver synchrotron performance in a system that will turn cryo-SXT into an affordable, efficient laboratory tool, thus increasing the scope and throughput of possible research projects. The key to this is the development of a sufficiently bright and compact source of soft X-rays.

The key technology at the core of the SiriusXT instrument is a high-performance soft X-ray light source based on laser-produced plasma emission with the appropriate size, wavelength and brightness, combined with smart optics whose optical quality is not degraded by the debris generated by the plasma. This unique combination enables the deployment of a lab-scale stable and robust light source suitable for cryo-SXT. The technology works by focusing a high power pulsed laser onto a solid target made from appropriate metals, producing a tiny million degree plasma. This laser plasma is hot enough to emit soft X-rays sufficient for efficient cell imaging, but it also produces a lot of metal debris. Delicate optics are required to collect the soft X-rays for use in the microscope and for focusing the laser, and these would be very quickly destroyed by the debris from the plasma. The company has developed a self-healing liquid coating for both the soft X-ray and laser focusing optics, which is continuously replenished and means that the optics can last indefinitely even in these extreme conditions.

With a field of view of $\sim 10-20 \mu \mathrm{m} \mathrm{x} \sim 10-20 \mu \mathrm{m}$, a penetration depth of $\sim 10 \mu \mathrm{m}$ and a resolution of $\sim 30$ $\mathrm{nm} 3$, the soft X-ray microscope neatly fits between the imaging capabilities of light and electron microscopes. The cryo-SXT niche can be broadly divided into the following four categories [8]; complex 3D structures in whole cells (mitochondrial networks, nuclear morphology etc.), volatile structures that are difficult to capture with chemical fixatives (autophagosomes, tabulated endosomes etc.), samples where accurate volumetric measurements are important (organelle volumes, cell volumes, gross morphological changes due to drug treatment or disease etc.), and samples in which compositional information is relevant (iron, nanoparticles, metal labels etc.).

We present data on light source performance and first images from our microscope [10]. 


\section{References:}

[1] C.A. Larabell \& K.A. Nugent, Current Opinion in Structural Biology 20 (2010), p.623.

[2] G. Schneider et al, Nature Methods 7 (2010), p.985.

[3] R. Carzaniga et al, Protoplasma 251 (2014), p.449.

[4] J.L. Carrascosa et al, Journal of Structural Biology 168 (2009), p.234.

[5] B.P. Cinquin et al, Journal of Cellular Biochemistry 115 (2014), p.209.

[6] R. Carzaniga et al, Methods in Cell Biology 124 (2014), p.151.

[7] K.C. Dent et al, Methods in Cell Biology 124 (2014), p.179.

[8] E. Duke et al, Journal of Microscopy 255 (2014), p.65.

[9] E.A. Smith et al, Biophysical Journal 107(2014), p.1988.

[10] The authors would like to thank the staff of the UCD School of Physics and Mechanical Workshop, for their continued contribution to this effort. In addition, we would like to thank Prof. Carolyn Larabell (NCXT, Berkeley), Dr. Lucy Collinson (The Francis Crick Institute, London), Dr. Eva Pereiro (ALBA, Barcelona) and Prof. Gerd Schneider (HZB, Berlin) for their support and advice, on a range of aspects including microscope design, implementation and help with defining biological applications of soft Xray tomography.

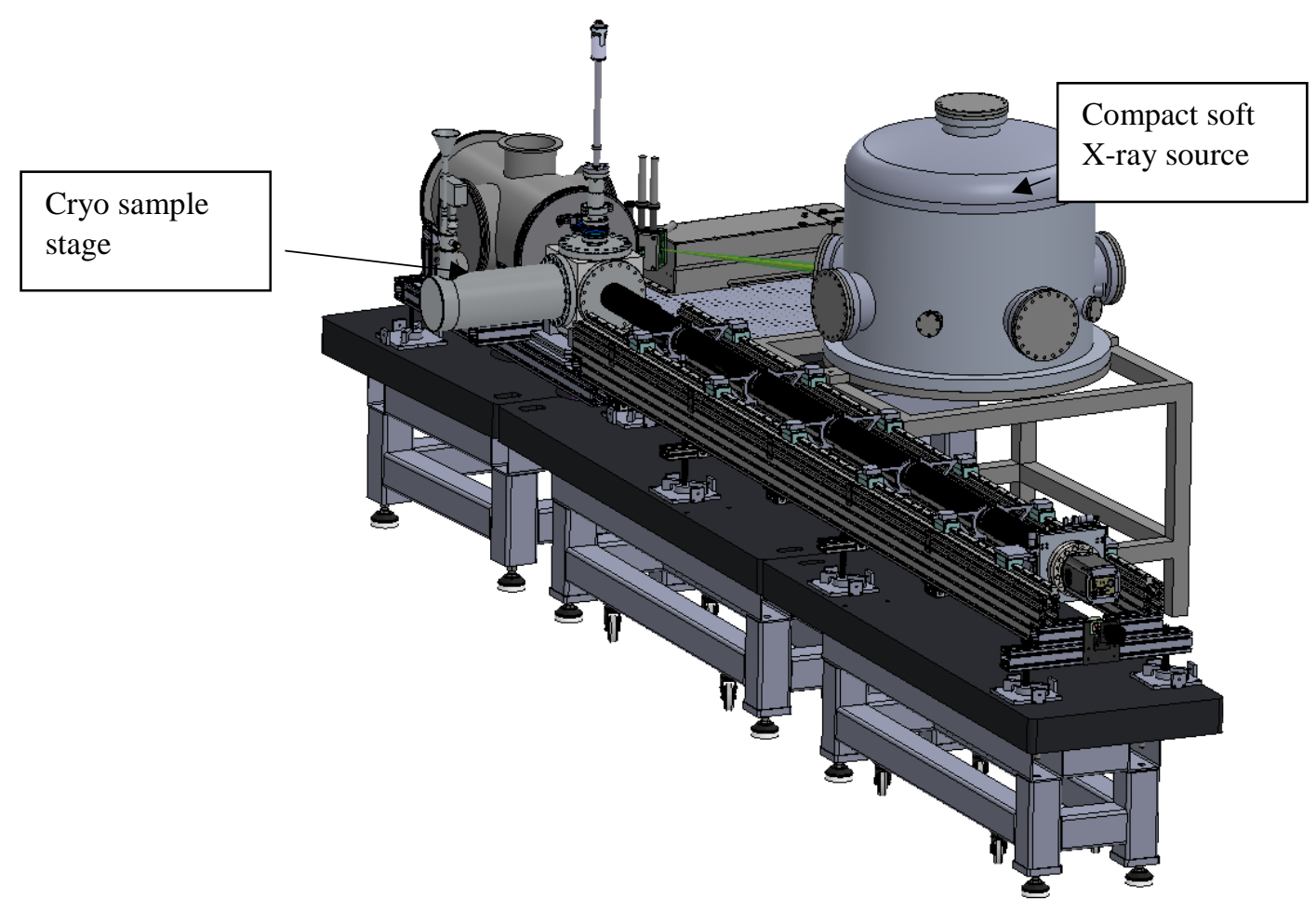

Figure 1. Conceptual design of the compact soft X-ray source and microscope. 